\title{
Effect of Supplemental Oxygen on Reinitiation of Breathing after Neonatal Resuscitation in Rat Pups
}

\author{
G. BRADLEY BOOKATZ, CATHERINE A. MAYER, CHRISTOPHER G. WILSON, MAXIMO VENTO, STEVEN L. GELFAND \\ MUSA A. HAXHIU, AND RICHARD J. MARTIN
}

\author{
Department of Pediatrics [G.B.B., C.A.M., C.G.W., S.L.G., M.A.H., R.J.M.], Rainbow Babies and Children's Hospital, Cleveland, Ohio \\ 44106; Servicio de Neonatología [M.V.], Hospital Universitario Materno-Infantil La Fe, 46009 Valencia, Spain
}

\begin{abstract}
To test our hypothesis that resuscitation with $21 \%$ and $40 \%$ oxygen $\left(\mathrm{O}_{2}\right)$ would shorten time to onset of respiratory activity when compared with resuscitation with $100 \% \mathrm{O}_{2}$, diaphragmatic electromyogram (EMG) electrodes were inserted in Sprague-Dawley rat pups, age $8-10 \mathrm{~d}$ before intubation and mechanical ventilation with $5 \% \mathrm{O}_{2}$ to induce cessation of respiratory activity. Each animal was then resuscitated with $100 \%$ and $21 \% \mathrm{O}_{2}(n=10)$ or $100 \%$ and $40 \% \mathrm{O}_{2}(n=11)$ for $30 \mathrm{~s}$ before the ventilator was disconnected. Recovery of diaphragm activity was compared between resuscitation groups. Blood gas status and heart rate data were characterized in additional rat pups. Time to first respiratory effort was $36 \pm 21 \mathrm{~s}$ $\left(\right.$ mean $\pm \mathrm{SD}$ ) for room air resuscitation and $72 \pm 22 \mathrm{~s}$ for $100 \% \mathrm{O}_{2}$, $(p=0.002)$. In contrast, there was no difference in time to onset of diaphragm activity when resuscitation with $40 \% \mathrm{O}_{2}$ was compared with $100 \% \mathrm{O}_{2}: 84 \pm 27 \mathrm{~s}$ versus $76 \pm 23 \mathrm{~s}$, respectively $(p>0.05)$. Resuscitation with $100 \%$ and $40 \% \mathrm{O}_{2}$ both resulted in hyperoxia and hypocapnia when compared with room air, without effect on heart rate. Our findings indicate that even modest hyperoxic resuscitation will result in a delayed onset of respiration compared with normoxic gas, via a mechanism that may involve both hyperoxemic and hypocapnic inhibition of chemoreceptors. (Pediatr Res 61: 698-702, 2007)
\end{abstract}

$\mathrm{T}$ The transition from fetal to neonatal life is frequently accompanied by a need for resuscitation with assisted ventilation. Guidelines issued by the American Academy of Pediatrics for neonatal resuscitation have traditionally recommended the use of $100 \% \mathrm{O}_{2}(1)$. Over the past two decades, the presumed benefits of pure $\mathrm{O}_{2}$ under these conditions have begun to be challenged. Saugstad and colleagues (2) were the first to show that use of room air was as effective as $100 \% \mathrm{O}_{2}$ in securing the survival of severely asphyxiated newborns while suggesting that room air might not possess the potentially dangerous side effects that $100 \% \quad \mathrm{O}_{2}$ presented. The potential dangers of $100 \% \mathrm{O}_{2}$ are illustrated in data of Vento et al. (3), showing that hyperoxic exposure following hypoxia causes greater oxidative stress than the use of a normoxic gas mixture. Recent recommendations are proposing a role for resuscitation with less than $100 \% \mathrm{O}_{2}$, and intermediate values

Received June 28, 2006; accepted January 29, 2007.

Correspondence: Richard J. Martin, M.D., Rainbow Babies and Children's Hospital, Division of Neonatology, 11100 Euclid Avenue, Cleveland, OH 44106-6010; e-mail: rxm6@case.edu

Supported by National Institutes of Health grant number HL 62527.

DOI: $10.1203 /$ pdr.0b013e3180534171 are being discussed, although the practical aspects and functional consequences remain to be determined (4).

To date, studies addressing the effect of assisted ventilation with supplemental $\mathrm{O}_{2}$ on the onset of respiration have been limited to trials in human neonates, such as the RESAIR 2 study (2). Such human studies have shown a delayed onset of both first cry and sustained respiratory effort when resuscitation is performed with $100 \% \mathrm{O}_{2}(3,4)$. To our knowledge, there have been no animal studies investigating the time to onset of spontaneous respiration following central apnea induced by hypoxic depression of breathing. Such investigation would provide more quantifiable data and eliminate some of the restrictions that accompany clinical trials. Piglets have been used to evaluate the effects of hyperoxic exposure on molecular and biochemical markers of lung and brain injury $(5,6)$. However, the piglet model presents limitations, in particular its relative maturity at birth and difficulty evaluating longer-term outcome measures. Therefore, in this study we have developed a rat pup model to test the efficacies of $21 \%$ and $40 \% \mathrm{O}_{2}$ against that of $100 \% \mathrm{O}_{2}$ in reinitiation of spontaneous respiratory activity following hypoxia induced apnea. We hypothesized that both $21 \%$ and $40 \% \mathrm{O}_{2}$ would shorten the time to onset of spontaneous respiratory activity when compared with resuscitation with the traditional standard of $100 \% \mathrm{O}_{2}$.

\section{METHODS}

Animal model and experimental protocol. The present study was conducted on Sprague-Dawley rats, 8-10 d old (22 g) and the protocol was approved by the Animal Care and Use Committee of Case Western Reserve University School of Medicine. The rat pups were anesthetized using urethane at a dosage of $2.5 \mathrm{~g} / \mathrm{kg}$ delivered via intraperitoneal injection, and then immobilized in the supine position. Urethane was used because it does not significantly depress central respiratory drive. Stainless steel EMG electrodes ( $0.13 \mathrm{~mm}$ bare, $0.18 \mathrm{~mm}$ coated) were then inserted through the abdomen to record diaphragmatic activity.

The study protocol is shown in Figure 1. All pups were orally intubated using an endotracheal tube (inner diameter, $0.76 \mathrm{~mm}$; outer diameter, 1.22 $\mathrm{mm})(7)$. After a brief period of mechanical ventilation to ensure adequate tube placement, the endotracheal tube was disconnected from the ventilator and baseline respiratory activity was recorded. The endotracheal tube was then reconnected to the ventilator, and the animals were ventilated with 5\% $\mathrm{O}_{2}$ (volume, $0.1 \mathrm{~mL} / 10 \mathrm{~g}$; rate, 35 cycles $/ \mathrm{min}$ ) until apnea was induced, as determined by a cessation of diaphragmatic activity. Ventilation with $5 \% \mathrm{O}_{2}$ was then continued for an additional $90 \mathrm{~s}$ after induction of apnea. At this

Abbreviations: EMG, electromyogram; $\mathbf{P a O}_{2}$, arterial oxygen tension; $\mathrm{PaCO}_{2}$, arterial carbon dioxide tension 


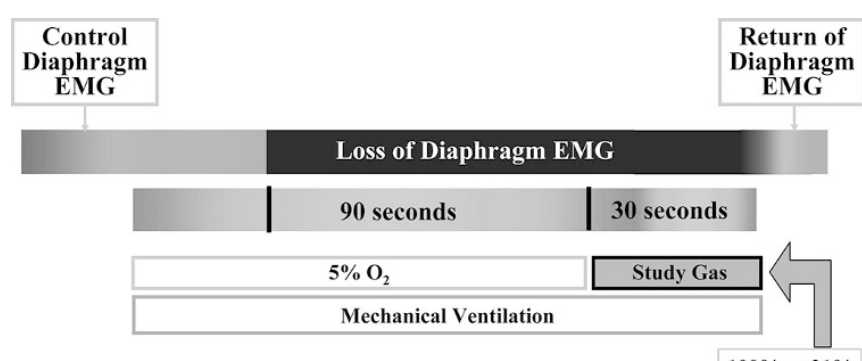

$100 \%$ vs $21 \%$

$100 \%$ vs $40 \%$

Figure 1. Experimental design. During the control period, the pups were intubated but not connected to a ventilator. During mechanical ventilation, the pups were initially ventilated with $5 \% \mathrm{O}_{2}$ to induce apnea, as illustrated by the cessation of diaphragmatic activity. They continued to be ventilated with 5\% $\mathrm{O}_{2}$ for an additional $90 \mathrm{~s}$ and then were resuscitated with the study gas for $30 \mathrm{~s}$. The ventilator was then disconnected and the return of diaphragm EMG quantified.

point, the ventilation gas was switched from $5 \% \mathrm{O}_{2}$ to study gas comprising either $100 \% \mathrm{O}_{2}$ or $21 \% \mathrm{O}_{2}(n=10)$ for $30 \mathrm{~s}$ at a ventilator rate of $60 / \mathrm{min}$. This period of study gas administration constituted the resuscitation period (Fig. 1). Following the $30 \mathrm{~s}$ resuscitation period, the ventilator was disconnected during the recovery period and onset of spontaneous diaphragmatic activity was quantified. Time to onset of spontaneous diaphragmatic activity was measured from the beginning of the resuscitation period. Following 45 min after the onset of the first breath, the identical protocol was performed with the alternate gas. The principal investigator was not blinded to the resuscitation gas, however, a standardized protocol of ventilatory management was systematically followed. In successive animals, the order of exposure to the resuscitation gas ( $100 \%$ versus $21 \%$ ) was alternated to compensate for any potential decline in the animals condition over the course of the experiment. The same protocol was used to compare $100 \% \mathrm{O}_{2}$ and $40 \% \mathrm{O}_{2}$ in an additional group of rat pups $(n=11)$. Data were only evaluated in animals that survived both resuscitation protocols. Data from 3 of 24 animals were not analyzed as the rat pup did not survive the second resuscitation. Thus, data from 21 animals were available between the two study protocols $(100 \%$ versus $21 \% \mathrm{O}_{2}$ and $100 \%$ versus $40 \% \mathrm{O}_{2}$ ). Each of the three animals that died did so during or immediately after the second exposure, which constituted $100 \%$ in two and $21 \%$ in the third.

Additional animals were studied using the same experimental protocol as for the physiologic study to obtain blood gas data. After intubation, and before exposure to ventilation with hypoxic gas, an incision was made lengthwise over the trachea, the tissue was retracted, and the carotid artery was exposed. In six animals, blood gas samples were then collected following the $90 \mathrm{~s}$ of apnea that preceded resuscitation. In three other groups $(n=6$ for each group), blood gas samples were collected immediately following $30 \mathrm{~s}$ exposure to the study gas $\left(21 \%, 40 \%\right.$, or $\left.100 \% \mathrm{O}_{2}\right)$. Finally, a blood gas was additionally collected upon onset of respiratory activity after $21 \%$ and $100 \%$ $\mathrm{O}_{2}$ resuscitation from five and four pups, respectively. Each animal could only be used for a single blood gas sample.

In six animals, additional ECG electrodes were inserted on the chest to collect heart rate data during $100 \% \mathrm{O}_{2}$ and room air resuscitation. A baseline heart rate was established after intubation and before ventilation. Heart rate values were compared when apnea was induced, before resuscitation, immediately after resuscitation and following recovery.

Data collection and statistical analysis. Diaphragmatic EMG and ECG were recorded using PowerLab hardware and Chart software in real time at a 2000-Hz sampling rate. The signal band pass for EMG recording was $0.03-1$ $\mathrm{kHz}$ and for ECG recording was $0.01-1 \mathrm{kHz}$. Within the Chart software, instantaneous heart rate was determined using the cyclic measurements tool. Data were analyzed using MatLab software for amplitude and frequency of diaphragmatic activity. Apnea was defined as an expiratory time longer than $15 \mathrm{~s}$. Onset of spontaneous respiratory activity was defined as the first diaphragmatic EMG that exhibited an amplitude of at least $10 \%$ of the diaphragmatic activity before hypoxic ventilation. We compared time to the onset of spontaneous diaphragm EMG between resuscitation with $100 \% \mathrm{O}_{2}$ and either $21 \%$ or $40 \% \mathrm{O}_{2}$ using a paired $t$ test.

Restoration of minute ventilation was also examined by computing minute diaphragm activity (diaphragm EMG $\times$ frequency per minute) as an index of minute ventilation. MatLab software was used to determine the area under individual bursts in the diaphragmatic EMG. We analyzed bursts over 5-s periods to determine the average burst at a given time frame. Sampling occurred at 30s, 60s, 90s, and $120 \mathrm{~s}$ following the onset of spontaneous respiration. By using the average burst and frequency data, we calculated minute diaphragm activity. Because the measured diaphragm activity varied on each animal due to the placement of EMG electrodes, the data were normalized by examining the restoration of minute ventilation as a percentage of the control (expressed as 100\%) before hypoxic ventilation for each animal. Two-way ANOVA with repeated measures was used to compare restoration of minute diaphragm activity between groups.

Blood gas samples collected via the carotid artery were analyzed using an ABL5 from Radiometer America (Westlake, $\mathrm{OH}$ ). Blood gas status in response to the various resuscitation gases was compared using one-way ANOVA and post hoc Newman-Keuls analysis.

Data collected by ECG electrodes were examined by comparing deviation from a baseline heart rate. One- and two-way ANOVA were used to determine whether heart rate differed between groups resuscitated with different $\mathrm{O}_{2}$ concentrations. All data in this manuscript are expressed as mean \pm SD.

\section{RESULTS}

All data were collected from animals that were exposed to both resuscitation gases comprising $100 \%$ and $21 \% \mathrm{O}_{2}$ or $100 \%$ and $40 \% \mathrm{O}_{2}$. We found a delayed onset of diaphragmatic discharge in rat pups resuscitated with $100 \% \mathrm{O}_{2}$ versus room air. Figure 2 shows the individual times to onset of breathing within each experimental group. Figure 3 presents summary data for the mean time to onset of spontaneous respiration in both experimental groups. A significant difference $(p=0.002)$ was found in the time to onset of spontaneous respiration between the room air ( $36 \pm 21 \mathrm{~s})$ and $100 \% \mathrm{O}_{2}$ $(72 \pm 22 \mathrm{~s})$ groups. This difference was apparently independent of the order of gas exposure. In contrast, there was no difference found between the use of $40 \% \mathrm{O}_{2}(84 \pm 27 \mathrm{~s})$ and $100 \% \mathrm{O}_{2}(76 \pm 23 \mathrm{~s})$.

In addition to onset of spontaneous respiratory activity, we compared the restoration of minute ventilation once sponta-
A

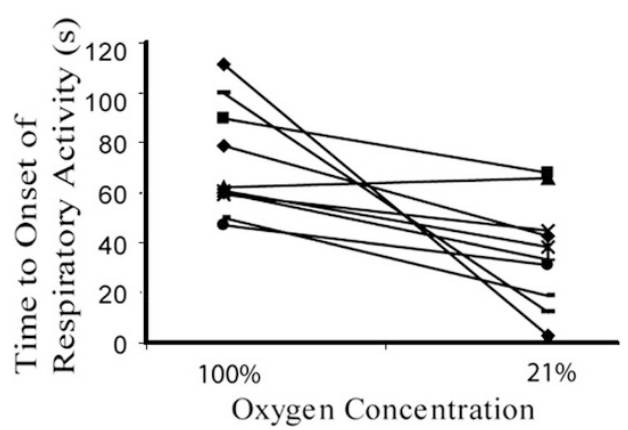

B

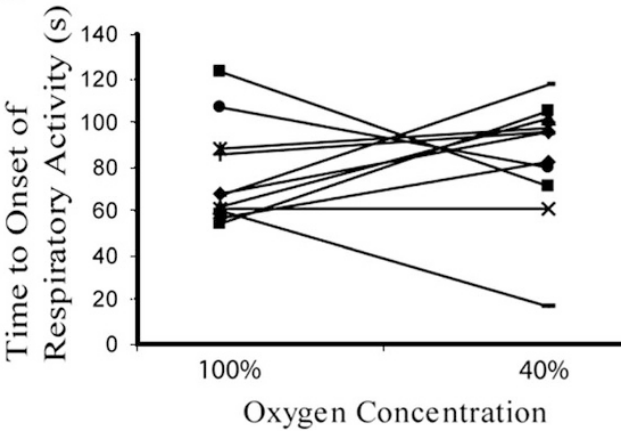

Figure 2. Individual results of onset of respiratory effort. Time to onset of diaphragm EMG during resuscitation with study gas is shown for each pup. (A) Onset of respiratory activity was delayed when $100 \% \mathrm{O}_{2}$ was compared with $21 \% \mathrm{O}_{2}(p=$ $0.002), n=10$. (B) In a separate group of rat pups, onset of respiratory activity was comparable between resuscitation with $100 \% \mathrm{O}_{2}$ and $40 \% \mathrm{O}_{2}, n=11$. 


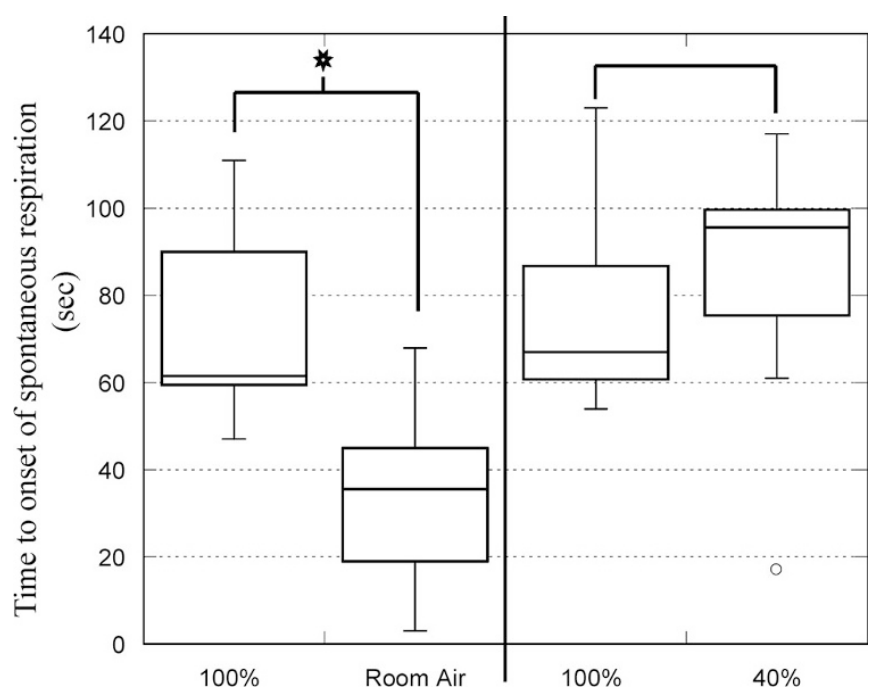

Figure 3. Grouped results of time to onset of respiratory effort. Summary data for time to onset of spontaneous respiration between experimental groups comparing $100 \% \quad \mathrm{O}_{2}$ vs $21 \% \mathrm{O}_{2}$ and $100 \% \mathrm{O}_{2}$ vs $40 \% \mathrm{O}_{2}$. *Significant difference $(p=0.002)$ in comparing the $100 \% \mathrm{O}_{2}$ group against $21 \% \mathrm{O}_{2}, n=$ 10. There was no difference between $100 \% \mathrm{O}_{2}$ and $40 \% \mathrm{O}_{2}, n=11$.

neous respiratory activity occurred. While room air was more effective in initiating onset of respiration there was no significant difference in restoration of minute ventilation between experimental groups ( $100 \%$ versus $21 \%$ or $100 \%$ versus $40 \%$ ) once diaphragmatic activity began (Fig. 4).

Arterial blood gas samples were collected at two points in the protocol; immediately following $90 \mathrm{~s}$ of apnea, before exposure to any of the study gases, and immediately following $30 \mathrm{~s}$ of exposure to each study gas. Blood gas status before resuscitation was as follows: $\mathrm{pH}=7.45 \pm$ $9 \times 10^{-9}\left[\mathrm{H}^{+}\right]$, arterial oxygen tension $\left(\mathrm{PaO}_{2}\right)=26 \pm 3 \mathrm{~mm} \mathrm{Hg}$, arterial carbon dioxide tension $\left(\mathrm{PaCO}_{2}\right)=30 \pm 7 \mathrm{~mm} \mathrm{Hg}, \mathrm{O}_{2}$ saturation $=51 \pm 10 \%$ and bicarbonate $=20 \pm 2 \mathrm{mM}$. Comparison of blood gas status after $30 \mathrm{~s}$ exposure to each study gas is shown in Table 1. As expected, there was an overall increase in $\mathrm{PaO}_{2}\left(p<0.0001\right.$, ANOVA) and $\mathrm{SaO}_{2}(p<$ 0.0001 , ANOVA) corresponding to the increase in $\mathrm{O}_{2}$ concentration of the study gas (Table 1). There was no effect of supplemental $\mathrm{O}_{2}$ on $\mathrm{pH}$, however, there was a decrease in $\mathrm{PacO}_{2}(p<0.05$, ANOVA $)$ and bicarbonate $(p<0.05$, ANOVA) in pups resuscitated with supplemental $\mathrm{O}_{2}$. $\mathrm{PaO}_{2}$ and $\mathrm{PaCO}_{2}$ data were also obtained at onset of spontaneous respiratory activity after resuscitation with $100 \% \mathrm{O}_{2}(n=4)$ and $21 \% \mathrm{O}_{2}(n=5)$. In the $100 \% \mathrm{O}_{2}$ group $\mathrm{Pao}_{2}=63 \pm 7$ $\mathrm{mm} \mathrm{Hg}, \mathrm{PaCO}_{2}=44 \pm 14 \mathrm{~mm} \mathrm{Hg}$ and in the $21 \% \mathrm{O}_{2}$ group $\mathrm{PaO}_{2}=66 \pm 10 \mathrm{~mm} \mathrm{Hg}, \mathrm{PaCO}_{2}=35 \pm 5 \mathrm{~mm} \mathrm{Hg}$.

We also chose to examine whether $100 \% \mathrm{O}_{2}$ and room air resuscitation had varying effects on heart rate. As seen in Figure 5, there was no change in heart rate from baseline during the experimental protocol (one-way ANOVA) and no difference between the $100 \% \mathrm{O}_{2}$ and room air groups (twoway ANOVA). Because no difference was found when comparing onset of respiration between the $40 \%$ and $100 \% \mathrm{O}_{2}$ groups, we did not examine heart rate data between these two groups.

\section{DISCUSSION}

Previous clinical studies have shown that when neonatal resuscitation is performed with room air, the time to onset of first cry and initiation of a sustained respiratory pattern are reduced when compared with the use of $100 \% \mathrm{O}_{2}(2,3)$. These findings are largely based on observational data rather than a quantifiable measure of respiratory activity. We therefore sought to develop an immature animal model that would allow us to quantify the effect of resuscitation gas at onset of spontaneous diaphragmatic activity. Our findings confirm those of available clinical studies comparing $100 \% \mathrm{O}_{2}$ versus room air resuscitation. In addition, we have examined an intermediate level of supplemental $\mathrm{O}_{2}, 40 \%$, compared its efficacy to that of the current standard of $100 \% \mathrm{O}_{2}$, and found no difference between the two gases with regards to onset of respiratory activity.

It is well known that neonatal exposure to hypoxia results in a biphasic respiratory response (8). This comprises an initial excitation of respiration secondary to excitation of peripheral chemoreceptors, followed by a central depression of respiration. We used exposure to $5 \% \mathrm{O}_{2}$ to elicit apnea in our model as low $\mathrm{O}_{2}$ tension probably contributes to inhibition of fetal respiratory activity and the respiratory depression during perinatal asphyxia. While hypoxic exposure always elicited apnea, there was variability in the time from onset of hypoxic exposure to onset of apnea. Therefore, we continued exposure with $5 \% \mathrm{O}_{2}$ for an additional $90 \mathrm{~s}$ to standardize the protocol between rat pups.
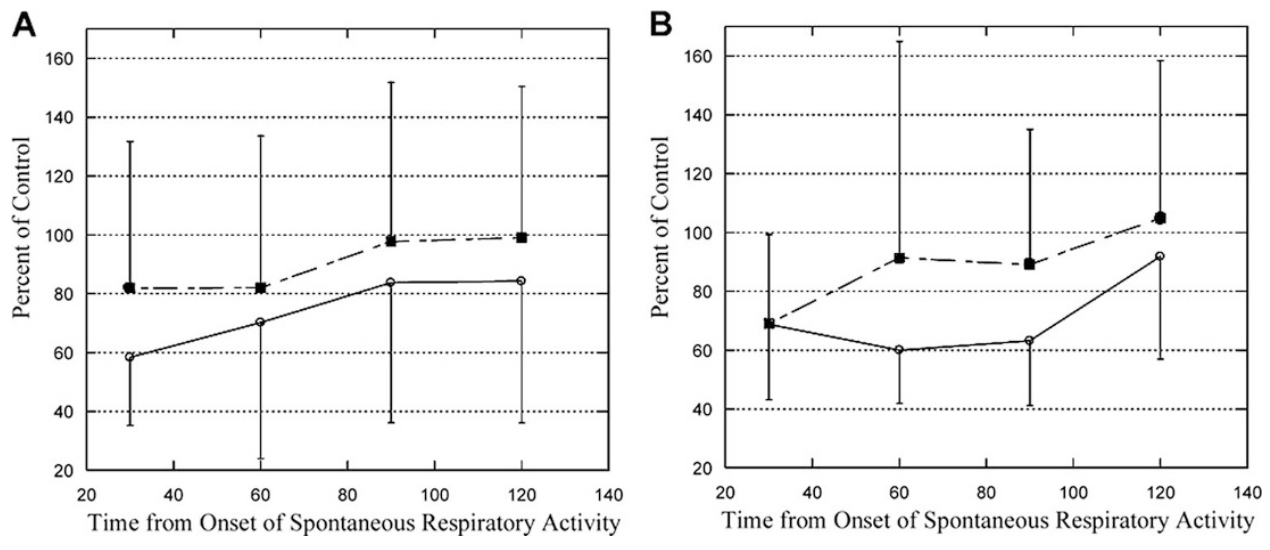

Figure 4. Restoration of minute ventilatory effort. Restoration of minute diaphragm activity did not differ when comparing (A) $100 \% \mathrm{O}_{2}$ vs $21 \% \mathrm{O}_{2}(n=10)$ and $(B)$ $100 \% \mathrm{O}_{2}$ vs $40 \% \mathrm{O}_{2}(n=11)$ resuscitation. There was considerable variability within the data as can be seen in the error bars that represent SD. Solid squares represent $21 \%$ and $40 \%$ oxygen, respectively; open circles represent $100 \%$ oxygen. 
Table 1. Blood gas status following 30 s resuscitation with study gas

\begin{tabular}{lccc}
\hline & $21 \% \mathrm{O}_{2}(n=6)$ & $40 \% \mathrm{O}_{2}(n=6)$ & $100 \% \mathrm{O}_{2}(n=6)$ \\
\hline $\mathrm{pH}$ & $7.37 \pm\left[1.15 \times 10^{-8}\right]$ & $7.45 \pm\left[9.26 \times 10^{-9}\right]$ & $7.37 \pm\left[5.12 \times 10^{-9}\right]$ \\
$\mathrm{Paco}_{2}(\mathrm{~mm} \mathrm{Hg})$ & $30 \pm 9.1$ & $19 \pm 6.5^{\dagger}$ & $21 \pm 3.5 \dagger$ \\
$\mathrm{PaO}_{2}(\mathrm{~mm} \mathrm{Hg})$ & $82 \pm 17$ & $169 \pm 23^{*}$ & $233 \pm 8^{*}$ \\
$\mathrm{SaO}_{2}(\%)$ & $95 \pm 2.7$ & $99 \pm 0.5^{*}$ & $100 \pm 0.4^{*}$ \\
$\mathrm{HCO}_{3}(\mathrm{mmol} / \mathrm{L})$ & $17 \pm 4 \ddagger$ & $14 \pm 4 \ddagger$ & $11 \pm 3 \ddagger$ \\
\hline
\end{tabular}

The results of blood gas samples taken following $30 \mathrm{~s}$ of exposure to the study gases are shown. $\mathrm{pH}$ values are presented with mean $\pm \mathrm{SD}$, where $\mathrm{SD}$ is presented in $[\mathrm{H}+]$.

$\dagger p<0.05$ via Newman Keuls analysis versus $21 \%$.

$* p<0.01$ via Newman Keuls analysis versus $21 \%$.

$\ddagger p<0.05$ via a single factor ANOVA between groups.

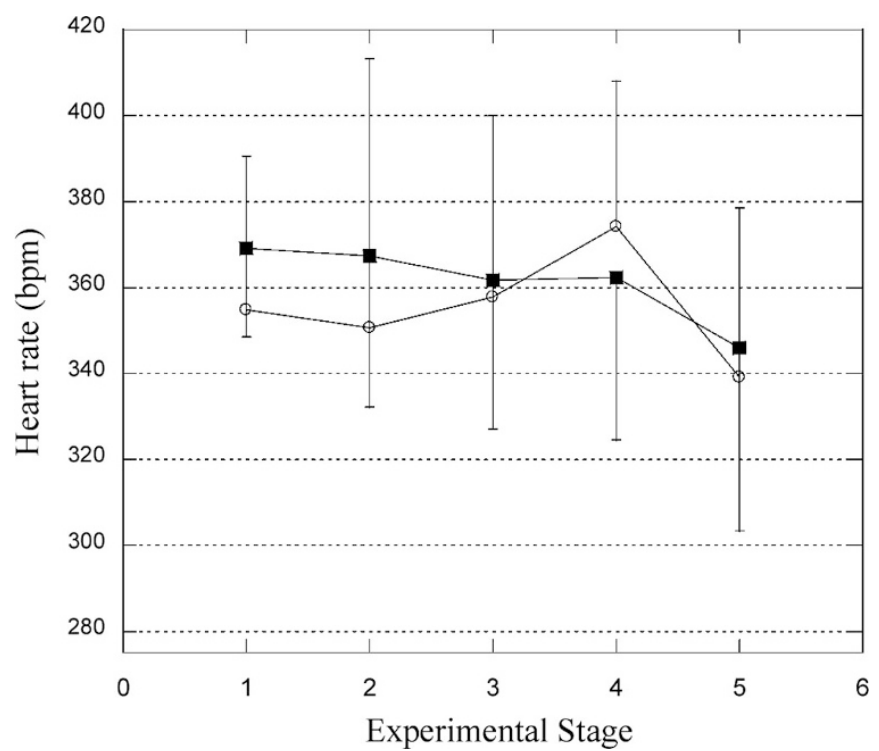

Figure 5. Heart rate activity during experiments. Average heart rate of the pups during the stages of the experiment in which heart rate was evaluated: (1) control, (2) onset of apnea, (3) before resuscitation, (4) following resuscitation, and (5) after recovery. There was no change in heart rate during the experiments or between groups. Solid squares represent $21 \%$ oxygen and open circles represent $100 \%$ oxygen.

During hypoxia, sustained increase in peripheral chemoreceptor drive will oppose central depression of respiration. Hence, hyperoxia during resuscitation should suppress peripheral chemoreceptor drive and delay reinitiation of breathing. This was first proposed by Dejours (9) and, more recently, adapted for the infant in studies by Bouferrache et al. (10). We hypothesized this to be a likely mechanism for the delayed onset of respiration when resuscitation with $100 \% \mathrm{O}_{2}$ is compared with the use of $21 \% \mathrm{O}_{2}$. Our blood gas results indicate that, as with $100 \% \mathrm{O}_{2}, 40 \% \mathrm{O}_{2}$ also creates a hyperoxemic environment, explaining the similar time to onset of respiratory activity when compared with $100 \% \mathrm{O}_{2}$. It is interesting to note quantitative similarities regarding onset of respiratory activity between the current data and human infant data from Saugstad and the RESAIR 2 study (2). Saugstad observed that the first breath occurred 1 min after beginning resuscitation with room air and after 1.5 min when $100 \%$ was used. We also found a difference of approximately $30 \mathrm{~s}$ between the onset of respiratory efforts when $21 \%$ and $100 \%$ $\mathrm{O}_{2}$ were compared. The fact that once initiation of respiratory activity began we found no difference in restoration of minute diaphragm activity leads us to believe that the difference found by Vento et al. (3) in time to sustained respiratory pattern may be attributed to the initial delay in onset of inspiration and not the recovery of rhythmic breathing.

Factors other than blood gas status (e.g. sensory stimuli or chemical mediators) have been proposed to contribute to onset of continuous breathing after birth (9). Jansen et al. (11) found that fetal carotid chemoreceptors are not essential for the establishment of effective breathing at birth in the lamb. Similarly, Hertzberg et al. (12) showed that arterial chemoreceptors are active and responsive in the fetal lamb, but that their sensitivity is adjusted to the low fetal arterial $\mathrm{O}_{2}$ tension and that after birth the chemoreceptors become silent and gradually reset their sensitivity. While our blood gas data revealed an anticipated incremental increase in $\mathrm{PaO}_{2}$ with increasing supplemental $\mathrm{O}_{2}$ resuscitation, we did not predict that supplemental $\mathrm{O}_{2}$ during resuscitation would result in the observed decrease in $\mathrm{Paco}_{2}$. This may occur via more rapid relief of pulmonary vasoconstriction in a hyperoxic environment and enhanced $\mathrm{CO}_{2}$ elimination, although the effect of hyperoxic resuscitation on pulmonary artery function remains controversial $(13,14)$. Whatever the cause, the possibility that hypocapnia, during resuscitation with supplemental $\mathrm{O}_{2}$, contributed to the delayed onset of breathing cannot be excluded as hyperoxemia and hypocapnia occurred in both groups resuscitated with supplemental $\mathrm{O}_{2}$. Further study is clearly appropriate to document whether hypocapnia is consistently associated with hyperoxic resuscitation. Such hypocapnia, if severe, could have potential detrimental effects on cerebral blood flow.

We recognize several reservations regarding our experimental model. Blood gases collected at the end of the apneic period before resuscitation were consistent with hypoxic respiratory depression but showed no evidence of metabolic or respiratory acidosis as one would expect in asphyxiated newborns. As the rat pups were continuously ventilated during hypoxic exposure, hypercapnia did not occur. This blood gas status was consistently observed in our animal model before resuscitation and should not have influenced our comparison of respiratory response to resuscitation with varying concentrations of $\mathrm{O}_{2}$. We did attempt to replicate $\mathrm{CO}_{2}$ accumulation by adding $\mathrm{CO}_{2}$ concentrations as low as $1 \%$ to the hypoxic gas that was used to induce apnea, however, use of a hypercapnic, hypoxic gas failed to induce apnea and, thus, we were unable to conduct our experiments. An alternate approach to normalize $\mathrm{PaCO}_{2}$ might have been to actually administer supplemental 
$\mathrm{CO}_{2}$ during resuscitation, however, this strategy is not consistent with current clinical practice. We speculate that the lower bicarbonate levels observed during hyperoxic resuscitation may be secondary to systemic vasodilatation and greater release of lactic acid from tissues previously deprived of $\mathrm{O}_{2}$.

Another limitation of our model was that the experiments were not performed during the actual transition from fetal to neonatal life. While we chose a rat pup model due to the animal's relative immaturity at birth, our experiments took place $8-10 \mathrm{~d}$ after birth at a time when the animal's maturation approaches term gestation in the human. It has previously been shown that the cerebral cortex of a 12-13-d-old rat pup has similar maturation as that of a full-term newborn human infant (15). This postnatal age was chosen because of our ability to readily intubate and ventilate the rat pups at and beyond $8 \mathrm{~d}$ of age. Future studies might focus on comparable resuscitation protocols in rat pups closer to the transition from fetal to neonatal life.

Although our primary goal was to determine the effect of resuscitation gas on the onset of respiration, we also examined heart rate response as an important component of the resuscitation response. We found no significant effect on heart rate between the two resuscitation gases examined, $100 \%$ and $21 \% \mathrm{O}_{2}$. This suggests that $21 \% \mathrm{O}_{2}$ is no less suitable for use during resuscitation, although the absence of significant bradycardia during hypoxic exposure limits any conclusions we can make. Ideally, we would have also measured cardiac output, however, due to the size of the animals and the invasive nature of the procedure, we determined that this procedure would have compromised our primary data collection.

We conclude that our novel rat pup model allows an accurate assessment of the relative efficacies of $100 \% \mathrm{O}_{2}, 40 \%$ $\mathrm{O}_{2}$, and room air in reinitiating respiratory efforts following apnea. Our studies show that, under conditions of mild hypocarbia, even modest levels of supplemental $\mathrm{O}_{2}$, such as $40 \%$ $\mathrm{O}_{2}$, cause hyperoxia and delay the onset of spontaneous respiratory efforts. The latter may be clinically relevant if it results in a prolonged duration of ventilatory support, potentially predisposing to morbidity associated with neonatal lung injury. The International Liaison Committee on Resuscitation recently concluded that there is currently not enough evidence to specify the concentration of $\mathrm{O}_{2}$ to be used during resuscitation (16). Our findings suggest that resuscitation with supplemental $\mathrm{O}_{2}$ may place infants in both a hyperoxemic and hypocapnic state, which may be detrimental to neonatal wellbeing. Because of this potential danger, studies such as the current one are therefore needed to further investigate the effects that varying levels of $\mathrm{O}_{2}$ concentration have on resuscitation efforts and the corresponding physiologic responses.

\section{REFERENCES}

1. American Academy of Pediatrics American Heart Association 2006 Use of resuscitation devices for positive pressure ventilation. In: Textbook of Neonatal Resuscitation, 5th Ed. American Academy of Pediatrics, Elk Grove Village, p 3-14

2. Saugstad OD, Rootwlet T, Aalen O 1998 Resuscitation of asphyxiated newborn infants with room air or oxygen: an international controlled study. Pediatrics 102:e1

3. Vento M, Asensi M, Sastre J, Lloret A, García-Sala F, Pallardó FV, Vina J 2001 Resuscitation with room air instead of $100 \%$ oxygen prevents oxidative stress in moderately asphyxiated term neonates. Pediatrics 107:642-647

4. Martin RJ, Walsh MC, Carlo WA 2005 Reevaluating neonatal resuscitation with 100\% oxygen. Am J Respir Crit Care Med 172:1360-1361

5. Kondo M, Itoh S, Isobe K, Kondo M, Kunikata T, Imai T, Onishi S 2000 Chemiluminescence because of the production of reactive oxygen species in the lungs of newborn piglets during resuscitation periods after asphyxiation loads. Pediatr Res 47:524-537

6. Munkeby BH, Børke WB, Bjørnland K, Sikkeland LI, Borge GI, Halvorsen B, Saugstad OD 2004 Resuscitation with $100 \%$ O2 increases cerebral injury in hypoxemic piglets. Pediatr Res 56:783-790

7. Iben SC, Haxhiu MA, Farver CF, Miller MJ, Martin RJ 2006 Short-term mechanical ventilation increases airway reactivity in rat pups. Pediatr Res 60:136-140

8. Rigatto $\mathrm{H}$. Control of breathing in fetal life and onset and control of breathing in the neonate. In: Polin RA, Fox WW, Abman SH (eds) Fetal and Neonatal Physiology. Saunders, Philadelphia, 2004, pp 890-900

9. Dejours P 1963 Control of respiration by arterial chemoreceptors. Ann N Y Acad Sci 109:682-695

10. Bouferrache B, Filtchev S, Leke A, Marbaix-Li Q, Freville M, Gaultier C 2000 The hyperoxic test in infants revisited. Am J Respir Crit Care Med 161:160-165

11. Jansen AH, Ioffe S, Russell BJ, Chernick V 1981 Effect of carotid chemoreceptor denervation on breathing in utero and after birth. J Appl Physiol 51:630-633

12. Hertzberg T, Hellström S, Holgert H, Lagercrantz H, Pequignot JM 1992 Ventilatory response to hyperoxia in newborn rats born in hypoxia-possible relationship to carotid body dopamine. J Physiol 456:645-654

13. Fugelseth D, Børke WB, Lenes K, Matthews I, Saugstad OD, Thaulow E 2005 Restoration of cardiopulmonary function with $21 \%$ versus $100 \%$ oxygen after hypoxaemia in newborn pigs. Arch Dis Child Fetal Neonatal Ed 90:F229-F234

14. Lakshminrusimha S, Russel JA, Steinhorn RH, Ryan RM, Gugino SF, Morin FC 3rd, Swartz DD, Kumar VH 2006 Pulmonary arterial contractility in neonatal lambs increases with $100 \%$ oxygen resuscitation. Pediatr Res 59:137-141

15. Romijn HJ, Hofman MA, Gramsbergen A 1991 At what age is the developing cerebral cortex of the rat comparable to that of the full-term newborn human baby? Early Hum Dev 26:61-67

16. International Liaison Committee on Resuscitation 2005 International Consensus on Cardiopulmonary Resuscitation and Emergency Cardiovascular Care Science with Treatment Recommendations. Part 7: Neonatal resuscitation. Circulation 112:III-91III-99 